\title{
Research and Practice of "3D Deconstruction" in Sketch Teaching in Colleges and Universities
}

\author{
Xu Jichun, Hao Xiaohua \\ Guangzhou College of Technology and Business, Guangzhou, Guangdong, China
}

Keywords: fine arts education; modeling basis; sketch teaching; 3D demonstration; artistic design

\begin{abstract}
Sketch teaching is an elementary course in colleges and universities. Whereas, students have some deviation in the cognition of sketch due to longtime examination-oriented drills for college entrance examination. In this paper, it is proposed in longtime teaching practice that a teaching method of 3D deconstruction is used to manifest theories by real model and visual observation and establish a correct and systematic observation method by virtue of clay sculpture models and teaching aids before representing images, which has yielded good effects in practice and enabled students to improve their drawing standard.
\end{abstract}

\section{Introduction}

\subsection{Weak modeling of heads in sketch resulting from examination-oriented education} (highlighting surface but neglecting modeling)

China's present implementation of fine arts college entrance examination causes the rise of a huge examination-oriented after-school learning institution. Its mode is to formulate a full range of examination methods with regard to characteristics of fine arts college entrance examination. Such methods aim to seek quick success and benefit and enable students to improve drawing effects in a short time but fail to make students have a full understanding and merely cram them up with many models. As a result, students take little account of interest but attach great importance to format in sketch, whose drawings echo each other and show unitary techniques of drawing. Their models are discrete and have improvement in fine arts accomplishment.

\subsection{Pros and cons of unified examination and special enrollment of fine arts in college entrance examination}

Unified examination is arranged for the sake of the major of fine arts in all universities and colleges. Such a type of exams share something in common but have no characteristic. In such a type of exams, sketch of head is to draw a portrait by contrast to a photo; thus, schools are spared the model cost and save costs; the photo itself has fixed the head's light source, facilitating students to start withdrawing. However, its disadvantage is lack of students' improvisation. By improvisation, it means that when a real person is moving, one can capture the most moving characteristic. In contrast, special enrollment is an enrollment model adopted by some specialized or key universities and colleges, which is harder than the unified examination from where examinees are standing because the unified enrollment is concerned with drawing a photo but the special enrollment is concerned with drawing from life. In addition, it is targeted at only minority of students with a certain foundation of modeling ability.

\subsection{Students' deviation of cognition and dependency on inherent habits in college sketch teaching}

As the proverb goes, custom makes all things easy. Examinees usher in many different paradigms when they become college students. All the paradigms are picked up for the sake of college entrance examination. Some are good but some are bad. Such paradigms must be changed and sorted out, which is an essential task to all the students. Therefore, students must find out which problems they have before learning. First of all, students highlight surface effects but neglect real 
understanding while processing a portrait. Secondly, they fail to make a good composition or carefully examine whether they accurately draw models before yearning for effects in a hurry. How many chances are there for an architecture without a secure foundation to be presentable and solid? Thus, students' awareness and understanding about modeling must be corrected in this aspect.

\section{Construction of 3D Deconstruction Thought in Sketch}

In sketch teaching, head research starts from research on the abstract. In other words, heads of personalized characteristics shall be put aside in the first place. Instead, heads shall be considered to comprise the same surface pieces and have the same size. Each head shall be designed, constructed and weighed from the perspective of architecture. Hence, deconstruction of each head shall be typical. Firstly, a head shall be considered to be a cube rather than an ellipse or shape of a goose's egg in the brain, as a result of which, calculations can be simple and specific.

According to Bridgman's Constructive Anatomy, a head cube is about $15.24 \mathrm{~cm}$ wide, $20.32 \mathrm{~cm}$ tall and $19.05 \mathrm{~cm}$ thick from its front to the rear. Such measured values are drawn from measurement of a skull cube. Though part of underside is obstructed by neck, it is the base of skull seen from below the jaw and in the rear. Thus, any graph can be constructed on the "plane" resembling a square of cube. It is the theory of $3 \mathrm{D}$ deconstruction and observation.

\section{Teaching Method of 3D Deconstruction}

Then, how to quickly improve students' abilities to understand and model?

(1) Correct way to understand comes first. It refers to how to view, which has been well elaborated in Bridgman's Constructive Anatomy in the company of explanation with specific examples. Simply speaking, now that there is a 3D structure described in a specific method, any other explanation will not be required by logic; whereas, it is not the case in reality. Students are required to perceive the charming face and good figure of a beautiful lady as columns of stairs. Beyond doubt, it is a huge movement. Just ask someone who has never learned sketch to think this way and he/she will consider this to be a crazy idea. After all, to deconstruct a person is a method indeed, which, whereas, is an irregular cognition. Hence, it demands a process to recognize, which not all the students can grasp. For sure, it is a method of fine arts. Though Mr. Bridgman gives a thorough explanation, it will work better if turning Mr. Bridgman's legends to 3D teaching aids to gradually enable students to comprehend.

(2) Vivid interpretation and analysis guides students to make a correct choice. For instance, when someone is in front of choices at an intersection, it will save his/her time if being told a correct direction. Thus, a teacher's role is to tell students. And it is a teacher's responsibility to tell students which situation is in front of them, what they will experience and which thinking method they should attach importance to.

(3) 3D deconstruction is the most visual way to observe an model. Plane offers interpretation in only one angle but fails to repeatedly interpret the same object in multiple angles. For instance, the center line of head seems across the middle of nasal bone in the front but as the face turns, its center point crosses the philtrum at the bottom of nasal bone instead of nasal tip; two high points in the middle of brow ridge and two end points of lower jawbone echo each other to create a face surface, which are related to each other in all angles they are observed. Similarly, changes of facial features, expressions (ecstasy, anger, sorrow and joy), muscle groups and tissues cause changes of shape. Therefore, students can understand the internal cause of changes of head by deconstruction of bone and muscle tissues and real recurrence of the gradual square-to-round transition by clay sculpture.

\section{Observation Process and Practical Effects of Teaching Method of 3D Deconstruction}

(1) In the first place, it is necessary to give clarity to the primary and secondary in the 3D representation of head.

Head is considered to be a cube to differentiate some big surfaces. Hence, portrait, though 
complicated, is merely concerned with six big surface pieces as a whole, wherein, such big surfaces show three sense organs of the five sense organs of human body (eyes, nose and mouth). Such three organs are given a priority in the first place while the other two sides separately show one organ (ears). It is a regular way found among the common people. It also explains why students are obsessed with partial depiction. However, such three organs do not exist in isolation. It is supposed to draw a basic shape as a whole before depicting specific five organs. And the sequence one adopts in drawing directly determines which way of drawing he/she uses: way of drawing as a whole or way of going deeper partially.

(2) In order to have better understanding about method of shaping a head in representation, many correct observation methods may be adopted. For instance, superciliary arch and jaw may be regarded as a whole to check the perspective of jaw in drawing in which students often make mistakes. Besides, a small surface partitioned in sculpture is often found in the scrutinize from the superciliary arch. It is a surface on top of the superciliary arch and cheekbone in fact, along which turns between the other side of cheekbone and the light-and-shadow junction can be found. Both of the above confuse students often. Whereas, how such surfaces turn and split is clarified by clay sculpture, making students remember size and orientation of such surfaces. In addition, they see and take to such a visual observation method as soon as they see such changes.

(3) Some surfaces tend to create a a visual illusion. Whereas, 3D deconstruction in clay sculpture enables students to feel such changes by hand in real means, including changes between surfaces and 3D change and fusion. Specifically, big surfaces are presented in front of students to enable them to understand size, direction, and transition relationship of surfaces before being recovered to the look of a real person. As a result, students know not only the surface but also the nature and cause.

(4) The teaching model of demonstration by 3D deconstruction and variable experience of observation of shape do not bore students. Once students have some doubts about why and how to tackle, they will get an explanation with the presentation of a skull and portrait muscle model in which the skull is added with "eyes" (soil) and muscles are added with "skin" (soil). Upon such a presentation and explanation, many students will see and improve their ability of drawing a portrait beyond expectation.

\section{Conclusion}

The teaching method of 3D deconstruction in practice augments teaching effects and offers an enlightenment--it helps students more for enabling students to really understand the movement from skull to muscles and that from muscles to an intact portrait compared with boring theory interpretation, which is incomparable. Besides, the teaching method of 3D deconstruction somewhat helps students in developing a correct sketch observation method and improving their drawing. It is a responsibility of an art worker and teacher engaged in elementary teaching of sketch to know which beneficial attempt and exploration to be made on the path of modifying sketch teaching to better improve students' modeling ability.

\section{Acknowledgement}

Fund Project: 2015 Inter-disciplinary Construction Project in Guangdong "12th Five-Year Plan" of Philosophy and Social Science (GD15XYS10).

\section{References}

[1] Qie Liuyin. Brief Analysis on the Importance of Elementary Course of Sketch in the Major of Fine Arts in Colleges and Universities [J]. Art Education Research. March 25, 2018.

[2] Li Ji, Fu Qi. Research on Teaching Elementary Course of Sketch in the Major of Fine Arts and Painting [J]. Art Education Research. February 15, 2018.

[3] Ding Junjun. Research on Plane and 3D in Elementary Sketch Teaching [J]. Beauty \& Times (Midmonth). March 15, 2017. 